\title{
Frontal-Sensory Cortical Projections Become Dispensable for Attentional Performance Upon a Reduction of Task Demand in Mice
}

\section{OPEN ACCESS}

Edited by: Walter Adriani,

National Institute of Health (ISS), Italy

Reviewed by:

Brian N. Mathur,

University of Maryland, Baltimore,

United States

Todor Vassilev Gerdjikov,

University of Leicester

United Kingdom

*Correspondence:

Hirofumi Morishita

hirofumi.morishita@mssm.edu

Specialty section:

This article was submitted to

Decision Neuroscience,

a section of the journal

Frontiers in Neuroscience

Received: 13 September 2021 Accepted: 15 December 2021

Published: 11 January 2022

Citation:

Norman KJ, Bateh J, Maccario $P$,

Cho C, Caro K, Nishioka T, Koike H and Morishita H (2022)

Frontal-Sensory Cortical Projections Become Dispensable for Attentional Performance Upon a Reduction of Task Demand in Mice.

Front. Neurosci. 15:775256. doi: 10.3389/fnins.2021.775256

\author{
Kevin J. Norman 1,2,3,4,5, Julia Bateh ${ }^{1,2,3,4,5}$, Priscilla Maccario ${ }^{1,2,3,4,5}$, Christina Cho ${ }^{1,2,3,4,5}$, \\ Keaven Caro ${ }^{1,2,3,4,5}$, Tadaaki Nishioka ${ }^{1,2,3,4,5}$, Hiroyuki Koike ${ }^{1,2,3,4,5}$ and \\ Hirofumi Morishita ${ }^{1,2,3,4,5 *}$
}

\begin{abstract}
${ }^{1}$ Department of Psychiatry, Icahn School of Medicine at Mount Sinai, New York, NY, United States, ${ }^{2}$ Department of Neuroscience, Icahn School of Medicine at Mount Sinai, New York, NY, United States, ${ }^{3}$ Department of Ophthalmology, Icahn School of Medicine at Mount Sinai, New York, NY, United States, ${ }^{4}$ Mindich Child Health and Development Institute, Icahn School of Medicine at Mount Sinai, New York, NY, United States, ${ }^{5}$ Friedman Brain Institute, Icahn School of Medicine at Mount Sinai, New York, NY, United States
\end{abstract}

Top-down attention is a dynamic cognitive process that facilitates the detection of the task-relevant stimuli from our complex sensory environment. A neural mechanism capable of deployment under specific task-demand conditions would be crucial to efficiently control attentional processes and improve promote goal-directed attention performance during fluctuating attentional demand. Previous studies have shown that frontal top-down neurons projecting from the anterior cingulate area (ACA) to the visual cortex (VIS; ACAvIS) are required for visual attentional behavior during the 5choice serial reaction time task (5CSRTT) in mice. However, it is unknown whether the contribution of these projecting neurons is dependent on the extent of task demand. Here, we first examined how behavior outcomes depend on the number of locations for mice to pay attention and touch for successful performance, and found that the 2-choice serial reaction time task (2CSRTT) is less task demanding than the 5CSRTT. We then employed optogenetics to demonstrate that suppression ACAVIS projections immediately before stimulus presentation has no effect during the 2CSRTT in contrast to the impaired performance during the 5CSRTT. These results suggest that $A C A_{V I S}$ projections are necessary when task demand is high, but once a task demand is lowered, ACA $_{V I S}$ neuron activity becomes dispensable to adjust attentional performance. These findings support a model that the frontal-sensory ACAvIs projection regulates visual attention behavior during specific high task demand conditions, pointing to a flexible circuit-based mechanism for promoting attentional behavior.

Keywords: top-down projection, attention, task-demand, anterior cingulate cortex, visual cortex, mouse

\section{INTRODUCTION}

Top-down attention is a fundamental cognitive process that facilitates the detection of the most pivotal goal-directed stimuli from our dynamic environment. The frontal cortex, particularly the anterior cingulate cortex area (ACA), has been demonstrated to be a key mediator of top-down control of visual attention across species including rodents (Passetti et al., 2002; 
Chudasama et al., 2003; Pehrson et al., 2013; Kim et al., 2016; Koike et al., 2016). Among ACA neurons, recent studies in mice have demonstrated that frontal-sensory projections from the ACA to the primary visual cortex (VIS) (ACA $A_{V I S}$ ) modulate visual discrimination (Zhang et al., 2014; Moore and Zirnsak, 2017) and free-moving attentional behavior (Norman et al., 2021a) through modulation of visual cortex processing (Norman et al., 2021b). However, it is not known whether the contribution of this top-down circuit depends on task demand. A circuit mechanism capable of deployment under specific task-demand conditions would be crucial to efficiently control attentional processes and improve promote goal-directed attention performance during fluctuating attentional demand. Here, we aimed to determine how task demand impacts the contribution of frontal-sensory projection neurons to attentional behavior.

We hypothesized that ACAVIs projection neurons may only be necessary for visual attentional behavior under conditions of elevated task demand. We tested this hypothesis by subjecting mice to two tasks of varying task demand while selectively manipulating $\mathrm{ACA}_{\mathrm{VIS}}$ neural activity in mice. One way to impact the demand of anticipatory attention is to vary the number of locations for mice to scan and touch for successful performance. Here we used the tasks in which mice were required to sustain and divide their attention in anticipation of a random presentation of a brief stimulus at one location across select number of response windows; either 5 locations during the 5CSRTT (Carli et al., 1983) (5CSRTT, Supplementary Figure 1) or limited to 2 locations during the 2CSRTT (Dillon et al., 2009; van Gaalen et al., 2009). During these tasks, we employed optogenetics to suppress ACA $A_{V I S}$ projection activity during these tasks to assess to what extent task demand impacts the contribution of ACAVIS projection neurons to visual attentional behavior.

\section{MATERIALS AND METHODS}

\section{Experimental Model}

Adult, male C57Bl/6 mice (Charles River Laboratories, MA) were group-housed under a standard $12 \mathrm{~h}$ light/dark cycle in a temperature- and humidity-controlled vivarium. Training was initiated when mice were 9-10 weeks old. Mice were allowed access to water for $2 \mathrm{~h}$ each day and maintained approximately 85-90\% of their ad libitum weight during behavioral training. Food was available ad libitum throughout the experiment. All animal protocols were approved by the Institutional Animal Care and Use Committee at Icahn School of Medicine at Mount Sinai. Data of the 5CSRTT have been analyzed previously (Norman et al., 2021b), but only the mice which underwent both 5CSRTT and 2CSRTT with optogenetics (6 out of 8 mice) were reanalyzed in this study.

\section{Viral Strategies and Stereotaxic Procedures}

Following procedures previously described in Norman et al. (2021b), mice were anesthetized with $2 \%$ isoflurane and head-fixed in a mouse stereotaxic apparatus (Narishige, East Meadow, NY). AAV2-CamKII-eNpHR3.0-eYFP (UNC Viral Vector Core, Chapel Hill, NC) was injected bilaterally into the ACA. Bilateral ACA injection sites relative to Bregma area are: $\mathrm{AP}+0.7 \mathrm{~mm}, \mathrm{ML} \pm 0.2 \mathrm{~mm}, \mathrm{DV}-0.7 \mathrm{~mm} ; \mathrm{AP}+0.2$ $\mathrm{mm}, \mathrm{ML} \pm 0.2 \mathrm{~mm}, \mathrm{DV}-0.7 \mathrm{~mm}$; $\mathrm{AP}-0.3 \mathrm{~mm}, \mathrm{ML} \pm 0.2$ $\mathrm{mm}$, DV $-0.7 \mathrm{~mm}$. Bilateral VIS injection sites relative to lambda are: $\mathrm{AP}+0.0 \mathrm{~mm}, \mathrm{ML} \pm 3.0 \mathrm{~mm}, \mathrm{DV}-0.4 \mathrm{~mm}$; $\mathrm{AP}+0.1 \mathrm{~mm}, \mathrm{ML} \pm 2.85 \mathrm{~mm}, \mathrm{DV}-0.4 \mathrm{~mm}$; $\mathrm{AP}+0.1 \mathrm{~mm}$, $\mathrm{ML} \pm 3.15 \mathrm{~mm}, \mathrm{DV}-0.4 \mathrm{~mm}$. Each infusion (500 nl) was made at $150 \mathrm{nl} / \mathrm{min}$ using a microinjector set (Nanoject III) and glass pulled syringe. The glass pipettes $(1.14 \mathrm{~mm}$ outer diameter and $0.53 \mathrm{~mm}$ inner diameter, 3-000-203-G/X, Drummond Scientific, PA) were pulled on a P-97 Flaming/Brown type micropipette puller (Sutter Instrument, CA). The tip of the pulled glass pipettes was approximately $50 \mu \mathrm{m}$. The syringe was left in place for $1 \mathrm{~min}$ following the injection to reduce backflow of virus. Bilateral LEDs (Amuza, San Diego, CA) of $500 \mu \mathrm{m}$ diameter that delivered $470 \mathrm{~nm}$ light were implanted at the VIS. A glass cannula associated with LED was implanted at the region of interest, and was glued onto the brain and light from the LED traveled about $1 \mathrm{~mm}$ through glass cannula into the brain. Model info of the LED and light intensity info is also included. Behavioral testing occurred at least 3 weeks after viral injection to allow for maximal viral expression. The location of the cannula was mapped onto blank coronal slice templates taken from Paxinos and Franklin mouse brain atlas as previously described (Norman et al., 2021b). One mouse was excluded due to the off-target cannula implantation.

\section{Behavior}

\section{5- and 2-Choice Serial Reaction Time Task (5CSRTT/2CSRTT)}

\section{Apparatus}

5CSRTT and 2CSRTT (see also Supplementary Figure 1) was conducted in eight Bussey-Saksida operant chambers with a touchscreen system (Lafayette Instruments, Lafayette, IL) following procedures previously described in Norman et al. (2021b). Dimensions are as follows: a black plastic trapezoid [walls $20 \mathrm{~cm}$ high $\times 18 \mathrm{~cm}$ wide (at screen-magazine) $\times 24 \mathrm{~cm}$ wide (at screen) $\times 6 \mathrm{~cm}$ wide (at magazine)]. Stimuli were displayed on a touch-sensitive screen (12.1 inch, screen resolution $600 \times 800)$ divided into five response windows by a black plastic mask $(4.0 \times 4.0 \mathrm{~cm}$, positioned centrally with windows spaced $1.0 \mathrm{~cm}$ apart, $1.5 \mathrm{~cm}$ above the floor) fitted in front of the touchscreen. For the 5CSRTT, all five response windows were available, but during the 2CSRTT, three of the outer response windows were masked and only two adjacent center windows were available. Schedules were designed and data was collected and analyzed using ABET II Touch software (v18.04.17, Lafayette Instrument). The inputs and outputs of the multiple chambers were controlled by WhiskerServer software (v4.7.7, Lafayette Instrument).

\section{Habituation}

Following procedures previously described in Norman et al. (2021b), before 5CSRTT training, mice were first acclimated 
to the operant chamber and milk reward. The food magazine was illuminated and diluted (30\%) sweetened condensed milk (Eagle Brand, Borden, Richfield, $\mathrm{OH}$ ) was dispensed every $40 \mathrm{~s}$ after mice entered the food magazine. Mice needed to enter the reward tray 20 times during two consecutive $30 \mathrm{~min}$ sessions before advancing to the next stage. Mice were then trained to touch the illuminated response window. During this phase, a white square stimulus was presented randomly at one response window until it was touched. If the mouse touched the stimulus, the milk reward was delivered in conjunction with a tone and magazine light. Touches to non-stimuli had no consequence. After reaching criterion on this phase (20 stimulus touches in $30 \mathrm{~min}$ for 2 consecutive days), mice advanced to the 5CSRTT training phase.

\section{5-Choice Serial Reaction Time Task Training and Baseline}

Following procedures previously described in Norman et al. (2021b), mice were tested 5 days a week, 100 trials a day (or up to $30 \mathrm{~min})$. Each trial began with the illumination of the magazine light. After mice exited the food magazine, there was an intertrial interval (ITI) period of $5 \mathrm{~s}$ before a stimulus was presented randomly at one response window. If a mouse touched the screen during the ITI period, the response was recorded as premature and the mouse was punished with a $5 \mathrm{~s}$ time-out (house light on). After the time-out period, the magazine light illumination and house light switch off signaled onset of the next trial. After the ITI period, a stimulus appeared randomly in one of the five response windows for a set stimulus duration (this varied from 32 to $2 \mathrm{~s}$, depending on stage of training). A limited-hold period followed by the stimulus duration was $5 \mathrm{~s}$, during which the stimulus was absent but the mouse was still able to respond to the location. Responses during stimulus presence and limited holding period could be recorded either as correct (touching the stimulus window) or incorrect (touching any other windows). A correct response was rewarded with a tone, and milk delivery, indicated by the illumination of the magazine light. Failure to respond to any window over the stimulus and limitedhold period was counted as an omission. Incorrect responses and omissions were punished with a 5-s time-out. In addition, repeated screen touches after a correct or incorrect response were counted as perseverative responses. Animals started at stimulus duration of $32 \mathrm{~s}$. With a goal to baseline mice at a stimulus duration of $2 \mathrm{~s}$, the stimulus duration was sequentially reduced from $32,16,8,4$, to $2 \mathrm{~s}$. Animals had to reach a criterion $(\geq 50$ trials, $\geq 80 \%$ accuracy, $\leq 20 \%$ omissions) over 2 consecutive days to pass from one stage to the next. After reaching baseline criterion with the $2 \mathrm{~s}$ stimulus duration for 4 out of 5 days, mice began 5CSRTT testing.

\section{5-Choice Serial Reaction Time Task Testing}

Following procedures previously described in Norman et al. (2021b), during 5CSRTT testing, attention demand was increased by reducing and pseudo-randomly shuffling the stimulus duration to $2.0,1.5,1.0$, and 0.8 s for 4 out of 5 days. Following 5CSRTT testing, mice underwent stereotaxic viral surgery and then were reestablished to baseline criterion before optogenetic experiments (2.0 and $1.0 \mathrm{~s}$ stimulus duration). Between experimental testing days, mice were subjected to $2 \mathrm{~s}$ stimulus duration training to confirm that the mice maintain stable baseline criterion. Attention and response control were assessed by measuring the following performance: correct percentage $[(100 \times$ (correct responses)/(correct responses + incorrect responses + omissions)], percentage accuracy $[100 \times$ correct responses/(correct responses + incorrect responses)], percentage omission $[100 \times$ omissions/(omissions + correct responses + incorrect responses)], percentage of premature responses, percentage of perseverative responses, latency to correct response (s), and latency to reward collection (s) after correct choices. A premature response is a response given during the ITI before a stimulus appears. It is a measure of impulsive behavior. A perseverative response is when a response to any screen continues to be given after the correct or incorrect screen has been poked and before retrieval of the reward. It is a measure of compulsive behavior. Correct response latency (CRL) is the difference between the time of stimulus onset and the time of the correct response. It is a measure of attention and processing speed. Reward collection latency (RCL) is the difference between the time of a correct response and the time of reward collection and reflects motivation.

\section{2-Choice Serial Reaction Time Task Baseline and Testing}

Immediately following 5CSRTT testing, the mice underwent baselining for the 2CSRTT. During the 2CSRTT, 3 of the response windows were blocked and only 2 central response windows remained in which the stimulus could appear. Animals had to reach a baseline criterion ( $\geq 50$ trials, $\geq 80 \%$ accuracy, $\leq 20 \%$ omissions) with the $2 \mathrm{~s}$ stimulus duration at 1 of 2 locations for 4 out of 5 days to pass criteria before beginning 2CSRTT testing. During 2CSRTT testing, attention demand was increased by reducing and pseudo-randomly shuffling the stimulus duration to $2.0,1.5,1.0$, and $0.8 \mathrm{~s}$ for 2 consecutive days. A separate cohort of naïve mice underwent 2CSRTT training and testing, without prior 5CSRTT experience, using the same criteria as the 5CSRTT but with only 2 central response windows.

\section{Optogenetics}

Following procedures previously described in Norman et al. (2021b), optogenetic experiments were conducted using Teleopto system (Amuza, San Diego, CA). Transistor-transistor logic signals were sent to a signal generator (Rigol Technologies, Beijing, China) that drove the light at specific time points during the trials. During separate test sessions, bilateral blue LED cannula with $1 \mathrm{~mm}$ length of optic fiber and $1.3 \mathrm{~mm}$ for the center to center distance of optic fibers (TeleLCD-B-1000500-1.3, Amuza, San Diego, CA) were stimulated during either $-5:-2 \mathrm{~s}$ of ITI, $-3: 0 \mathrm{~s}$ of ITI or during the length of the stimulus presentation. One timing of stimulus duration was used per test day in a counterbalanced manner. During behavioral 5CSRTT and 2CSRTT, continuous $470 \mathrm{~nm}$ light was delivered bilaterally via LED optic fiber implanted at the VIS pseudo randomly at specific time points throughout a trial during 50\% of trials. In our study, we chose to use $470 \mathrm{~nm}$ LED to suppress eNpHR3.0-expressing ACA neurons, because previous studies found that red-shifted light frequencies resulted in off-target 
effects in control mice that reduced 5CSRTT performance (White et al., 2018). During optogenetics testing, stimulus duration was pseudo randomly shuffled between 2.0 and $1.0 \mathrm{~s}$. The power at the fiber-optic tip measured using an optical power meter (Thorlabs) was approximately $10 \mathrm{~mW}$.

\section{Quantification and Statistical Analysis}

Statistical analyses were performed using Prism (Graphpad, San Diego, CA) and in R v. 3.5.3. Analyses were conducted using general linear mixed models, ANOVA, or $t$-tests as indicated. Bar graphs are represented as the mean and error bars represent standard error of the mean (SEM).

\section{RESULTS}

\section{2-Choice Serial Reaction Time Task Shows Reduced Task Demand Compared to 5-Choice Serial Reaction Time Task}

We first sought to determine whether attentional behavior outcomes depend on the extent of task demand by manipulating a number of locations where visual stimulus is presented for mice to scan and touch to successfully obtain food reward. Specifically, we compared the performance between mice performing the 5CSRTT (Carli et al., 1983; Bari et al., 2008) and 2CSRTT with varying numbers of choice locations. Mice were first trained to perform the 5CSRTT (Figure 1A and Supplementary Figure 1A). The task required mice to sustain and divide their attention across 5 response windows in their lower visual field in anticipation of a brief white square stimulus appearing pseudorandomly at 1 of the 5 locations in automated standardized Bussey-Saksida touch-screen operant chambers (Bussey et al., 2012; Mar et al., 2013). The number of trials with correct touches to the flashed screen, compared to omitted and incorrect touches is a primary operational definition of attention. Correct response rate provides a broad and general index of attention performance and reflects what is captured by both accuracy and omission, two readouts that have been used to assess some aspects of attention in previous studies using 5CSRTT (Carli et al., 1983). It should be noted that omission probability is sensitive to both lapse in attention and motivation and every operant response depends to some degree on both. However, another readout of the 5CSRTT, the latency to collect reward following a correct response, serves as an indicator of motivation to assess if omission change reflects attention or motivation level. In addition, other 5CSRTT behavioral measures such as premature, and preservative response assess relatively independent indices of cognition (Dalley et al., 2004; Lustig et al., 2013; Koike et al., 2016; Supplementary Figure 1B). Mice had to reach baseline criteria of 4 out of 5 sessions with $\geq 50$ trials, $\geq 80 \%$ accuracy, $\leq 20 \%$ omission at $2 \mathrm{~s}$ stimulus duration before 5CSRTT testing in which the stimulus duration length was shuffled between $2.0,1.5,1.0$, and $0.8 \mathrm{~s}$ in a pseudorandomized order. Following 5CSRTT testing, mice began 2CSRTT baselining with $2 \mathrm{~s}$ stimulus duration in which the number of response windows was reduced to 2. To pass 2CSRTT baselining stage, mice had to reach same baseline criteria as the 5CSRTT before undergoing 2CSRTT testing. We found that task performance of mice was significantly higher during the 2CSRTT compared to the 5CSRTT as indicated by increased correct trials [Figure 1B: two-way repeated measures analysis of variance (RM ANOVA) task type $P=0.0059$ ]. The improved task performance during the 2CSRTT was driven by improved accuracy (Figure 1C: two-way RM ANOVA, task type $P=0.0118$ ) with a lesser degree of change in omissions (Figure 1D: two way RM ANOVA, task type $P=0.1146$ ). Furthermore, CRL was slower during the 5CSRTT (Figure 1E: $t$-test $P=0.0002$ ), while reward collection latency(s) (Figure 1F: $t$-test $P=0.7720$ ), premature responses (\%) (Figure 1G: $t$-test $P=0.3218$ ), and perseverative responses (\%) (Figure $\mathbf{1 H}$ : t-test $P=0.2377$ ) were not statistically different between both the 5CSRTT and 2CSRTT. These data suggests that each task engaged similar levels of motivation, impulsivity, and compulsivity, respectively.

Of note, there was no difference in 2CSRTT performance between mice with or without prior 5CSRTT testing (Figure 2). Specifically, neither correct\% (Figure 2B: two-way RM ANOVA, task type $P=0.9379$ ), accuracy\% (Figure 2C: two-way RM ANOVA, task type $P=0.9489$ ) nor omission\% (Figure 2D: two-way RM ANOVA, task type $P=0.9383$ ) was different during 2CSRTT between mice with or without prior 5CSRTT testing. No difference in CRL (Figure 2E: $t$-test $P=0.3119$ ), reward collection latency (Figure 2F: $t$-test $P=0.9363$ ), premature responses (Figure 2G: t-test $P=0.2913$ ), and perseverative responses (Figure $2 \mathrm{H}: P=0.1111$ ) was observed between the two groups. These results suggest that the order of training has limited influence to attentional behavior. Collectively, these data support that lower task demand of 2CSRTT compared to 5CSRTT is reflected to better task performance in 2CSRTT.

\section{ACA $_{\text {vIs }}$ Projections, While Required for 5-Choice Serial Reaction Time Task, Are Dispensable for 2-Choice Serial Reaction Time Task}

While our previous studies have shown that $\mathrm{ACA}_{\mathrm{VIS}}$ projection neurons are required for attentional behavior during 5CSRTT in mice (Norman et al., 2021a,b), it is unknown whether the contribution of this subpopulation of frontal cortex projecting neurons are task demand dependent. To this end, we selectively suppressed $\mathrm{ACA}_{\mathrm{VIS}}$ axon terminal activity in visual cortex using the inhibitory opsin halorhodopsin eNpHR3.0 at key time points during both the 5CSRTT and 2CSRTT in which the stimulus duration length was shuffled between 2 and $1 \mathrm{~s}$ in a pseudorandomized order to determine its effect on task performance (Figures 3A,B). Data of the 5CSRTT have been analyzed previously (Norman et al., 2021b), but only the mice which underwent both 5CSRTT and 2CSRTT (6 out of 8 mice) were re-analyzed in this study. Viral spread and implant location were validated and reported in a previous study that used the mice to this study (Norman et al., 2021b). This study also 


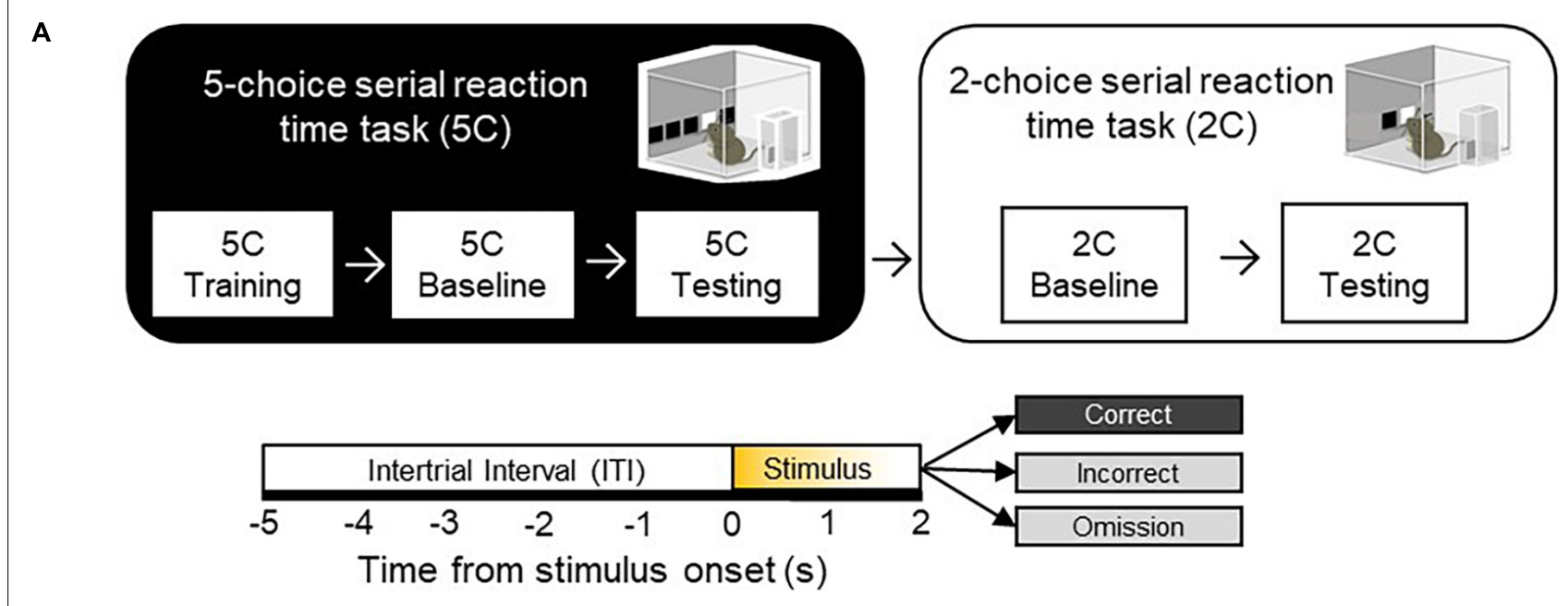

B

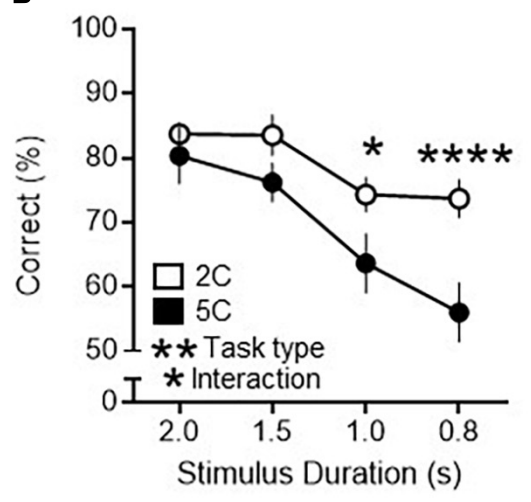

C

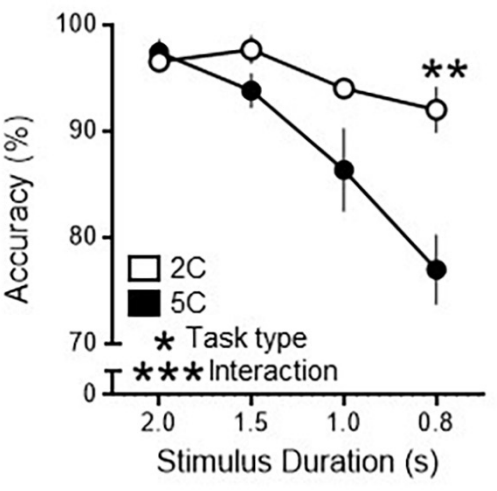

D

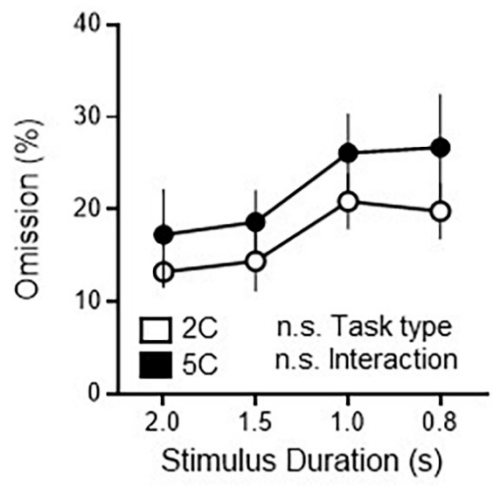

E

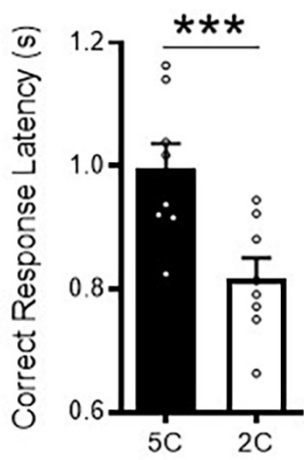

F

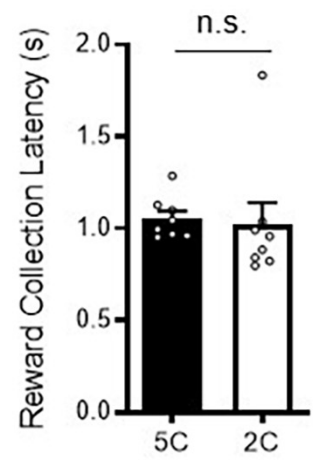

G

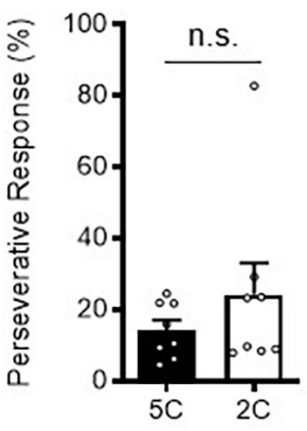

H

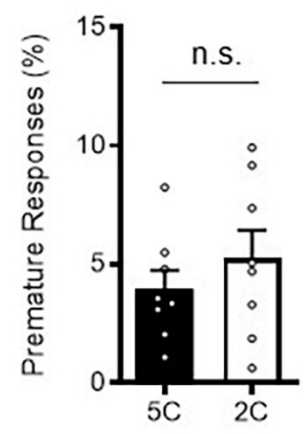

FIGURE 1 | 2CSRTT is less task demanding than the 5CSRTT. (A) Experimental timeline: Mice were first trained on the 5CSRTT before undergoing 5CSRTT testing During 5CSRTT testing, with a fixed $5 \mathrm{~s}$ ITI and pseudorandomized stimulus duration (2.0, 1.5, 1.0, or $0.8 \mathrm{~s})$, (8 mice; 2,161 total trials). The same mice were then baselined with 2CSRTT before undergoing 2CSRTT testing (2,354 total trials). (B) Mice had a significantly increased correct trials during the 2CSRTT compared to the 5 CSRTT (\%, two-way repeated measures analysis of variance (RM ANOVA), Main effect (task type) $F_{(1,7)}=15.26$, ${ }^{\star \star} P=0.0059$, Main effect (stimulus duration): $F_{(3,21)}=29.48, P<0.0001$, Interaction: $F_{(3,21)}=3.626$, ${ }^{\star} P=0.0298$, Holm-Sidak multiple comparisons at 2.0 , 1.5 , 1.0, 0.8 s stimulus duration, $P=0.7623,0.1197,{ }^{\star} 0.0121,{ }^{\star \star \star \star}<0.0001, n=8$ mice). (C) Mice had a significantly increased accuracy during the 2 CSRTT compared to the 5 CSRTT (\%, two-way RM ANOVA, Main effect (task type): $F_{(1,7)}=11.40,{ }^{\star} P=0.0118$, Main effect (stimulus duration): $F_{(1.53,10 \cdot 71)}=31.92, P<0.0001$, Interaction: $F_{(3}$, 21) $=10.64,{ }^{\star \star \star} P=0.0002$, Holm-Sidak multiple comparisons at 2.0, 1.5, 1.0, 0.8 s stimulus duration, $P=0.9781,0.2574,0.3198,{ }^{\star \star} 0.0017, n=8$ mice). (D) Mice had no difference in omissions during the 2CSRTT compared to the 5CSRTT (\%, two-way RM ANOVA, Main effect (task type): $F(1,7)=3.246, P=0.1146$, Main effect (stimulus duration): $F_{(3,21)}=9.526, P=0.0004$ Interaction: $F_{(3,21)}=0.2317, P=0.8733, n=8$ mice). (E) The 2CSRT had a significantly reduced correct response latency compared to the $5 \mathrm{CSRTT}\left(t_{7}=7.193,{ }^{\star \star \star} P=0.0002\right)$. (F-H) No difference in $(\mathbf{F})$ reward collection latency $\left(t_{7}=0.3012, P=0.7720\right),(\mathbf{G})$ premature responses $\left(t_{7}=1.066, P=0.3218\right)$, and $(\mathbf{H})$ perseverative responses $\left(t_{7}=1.291, P=0.2377\right)$ was observed between $2 \mathrm{CSRTT}$ and $5 \mathrm{CSRT}$ testing. Error bars indicate mean \pm SEM. n.s., non-significant. * indicates $P<0.05$, ${ }^{* \star}$ indicates $P<0.01,{ }^{\star \star \star}$ indicates $P<0.001,{ }^{\star \star \star \star *}$ indicates $P<0.0001$. 


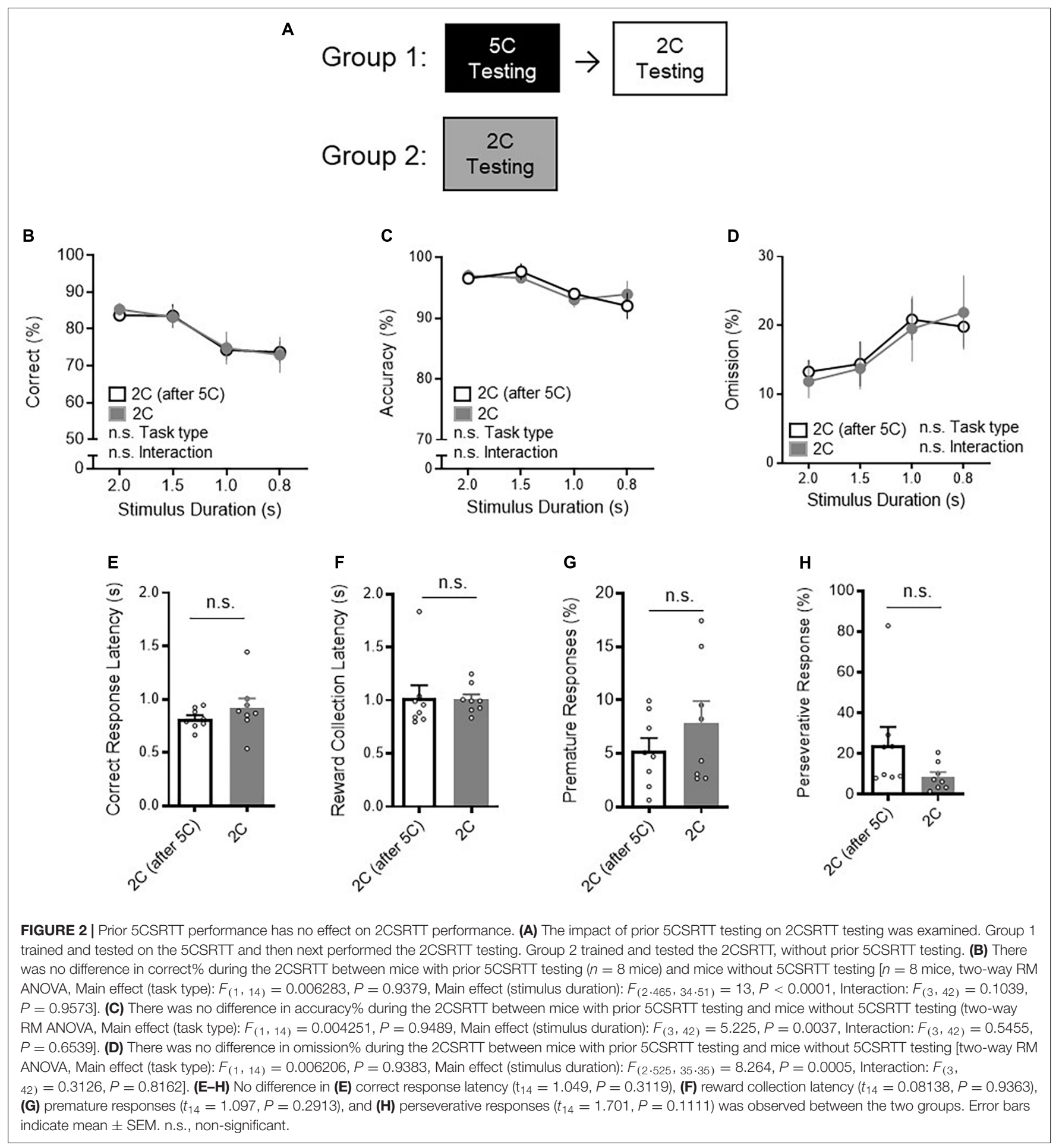

showed that patch-clamp recording from ACAvis neurons in slices effectively suppressed spike activities without rebound excitation (Norman et al., 2021b).

Correct response rate provides a broad and general index of attention performance and reflects what is captured by both accuracy and omission, two readouts that have been used to assess some aspects of attention in previous studies using 5CSRTT (Carli et al., 1983). We found that suppressing $\mathrm{ACA}_{\mathrm{VIS}}$ projections during the $3 \mathrm{~s}$ immediately before stimulus presentation (-3:0 s) impaired performance during the 5CSRTT, reducing correct trials [Figure 3F general linear mixed model (GLMM) effect of light during 5C task $P=0.0114$ ] by increasing omission (Figure 3G effect of light during 5C task $P=0.0001$ ), however, no effect was observed during the 2CSRTT 

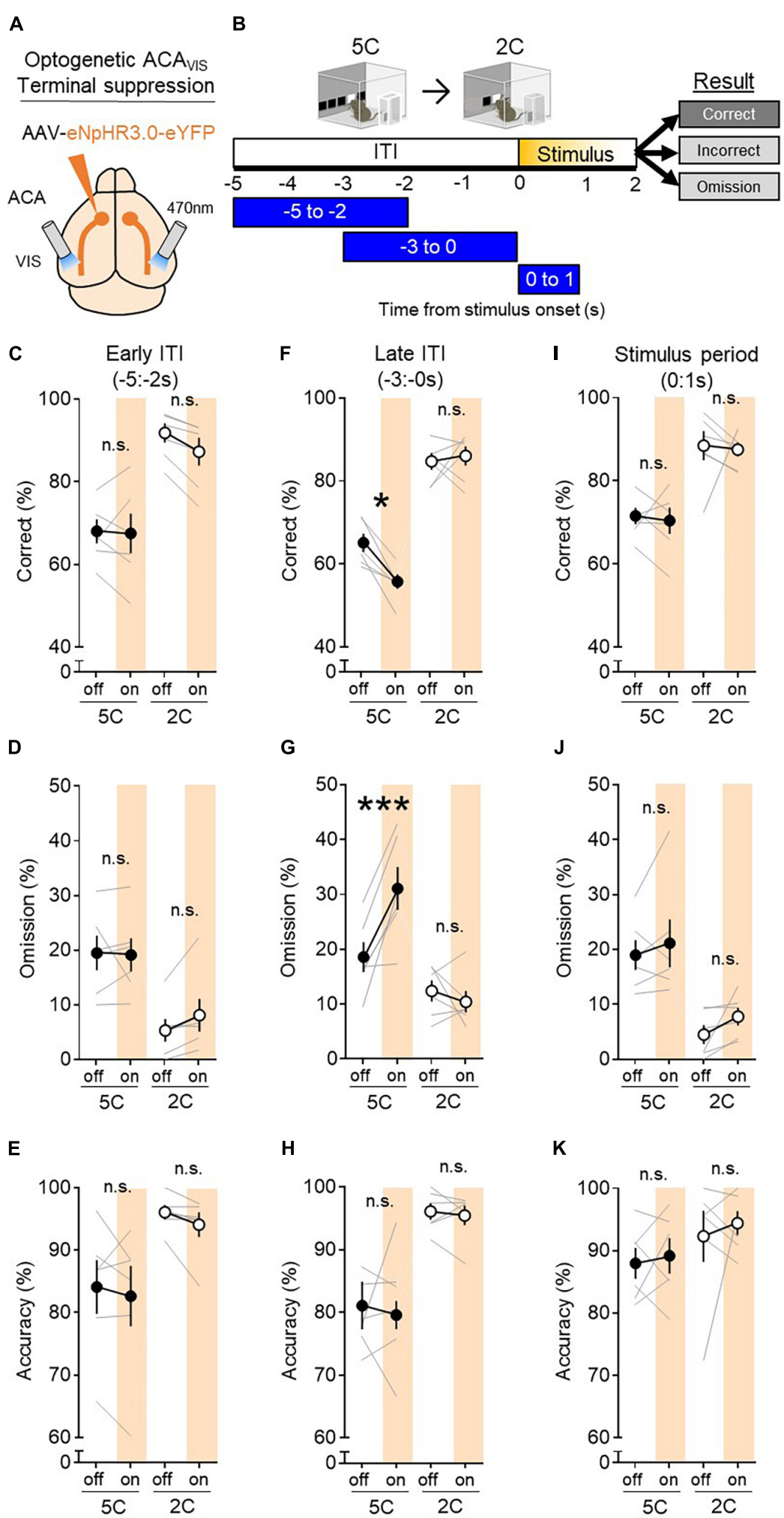

FIGURE 3 ACA $_{\text {VIS }}$ projections, while required for 5CSRTT, are dispensable for 2CSRTT. (A) Viral strategy: To express light-gated chloride channel eNpHR3.0 in excitatory ACA neurons, eNpHR3.0 encoding AAV under the CaMKII promoter was injected in the ACA. An optic fiber was implanted at the VIS to optically suppress $\mathrm{ACA}_{\mathrm{VIS}}$ projection terminals with temporal precision during the 5CSRTT. (B) Experimental timeline: Mice were first trained on the $5 \mathrm{CSRTT}$, before viral injection. After allowing 3 weeks for maximal viral expression, mice underwent 5CSRTT testing. During 5CSRTT testing, with a fixed $5 \mathrm{~s}$ ITI and pseudorandomized stimulus 
FIGURE 3 | duration (2.0 or $1.0 \mathrm{~s}), 470$ nm LED was illuminated during early ITI (-5:-2 s), late ITI (-3:0 s), or during period of stimulus presentation (0:1 s) during $50 \%$ of trials (6 mice; 2,694 total trials). One timing was tested per day. The effect of optogenetic suppression during early ITI, late ITI, or stimulus period on behavioral performance (correct\%, omission\%, and accuracy\%) at each light timing tested. The same mice were then baselined with 2CSRTT before undergoing the same optogenetic manipulation protocol with the 2CSRTT testing (6 mice; 2,844 total trials). (C-E) Continuous 470 nm illumination during ITI -5:-2 s had no effect on correct trials (\%, general linear mixed model (GLMM), effect of light: $P=0.1010$, effect of task: $P<0.0001$, interaction: $P=0.1412$ ), omissions (GLMM, effect of light $P=0.2873$, effect of task: $P<0.0001$, interaction: $P=0.2214)$, or accuracy (GLMM, effect of treatment (light on vs. off): $P=0.1796)$. (F-H) Continuous 470 nm illumination during ITI $-3: 0 \mathrm{~s}$ had an effect on correct trials (GLMM, effect of light: $P=0.1274$, effect of task: $P<0.0001$, interaction: $P=0.0305)$ and omissions (GLMM, effect of light $P=0.0470$, effect of task: $P<0.0001$, interaction: $P=0.0008$ ). Light disrupted correct trials during the $5 \mathrm{C}$ task $\left({ }^{\star} P=0.0114\right)$, but not during the $2 \mathrm{C}$ task $(P=0.9784)$. Light disrupted omissions during the $5 \mathrm{C}$ task ( $\left.{ }^{* \star *} P=0.0001\right)$, but not during the $2 \mathrm{C}$ task $(P=0.8267)$. Continuous $470 \mathrm{~nm}$ illumination during ITI $-3: 0$ s had no effect on accuracy (GLMM, effect of light: $P=0.6049$, effect of task: $P<0.0001$, interaction: $P=0.6068$ ). (I-K) Continuous 470 nm illumination during the stimulus period had no effect on correct trials (GLMM, effect of light: $P=0.4721$, effect of task: $P<0.0001$, interaction: $P=0.7455$ ), omissions (GLMM, effect of light: $\mathrm{P}=0.0242$, effect of task: $\mathrm{P}<0.0001$, interaction: $\mathrm{P}=0.1570$, effect of light during $5 \mathrm{C}$ task $\mathrm{P}=0.6911$, during $2 \mathrm{C}$ task $P<0.9702)$, or accuracy (GLMM, effect of light: $P=0.2600$, effect of task: $P=0.0009$, interaction: $P=0.5844)$. Error bars indicate mean $\pm S E M$. n.s., non-significant. * indicates $P<<0.05$, ${ }^{* \star}$ indicates $P<<0.001$.

(Figures 3F,G GLMM, effect of light during 2C task: correct $P=0.9784$, omission $P=0.8267)$. We did not observe effect of light illumination on accuracy (Figure 3H GLMM, effect of light $P=0.6049$ ). These findings indicate that it is mainly the omission increase that contributed to the correct rate reduction by the optogenetic suppression of ACAvis projections during 5CSRTT.

Performance of either task was not affected by adjusting the LED illumination time to earlier in the ITI $(-5:-2 \mathrm{~s})$ (Figures 3C-E: GLMM, effect of light: correct trials $\% P=0.1010$, omissions\% $P=0.2873$, accuracy\% $P=0.1796)$ or during stimulus presentation (0:1 s) (Figures 3I-K: GLMM, correct trials\%: effect of light $P=0.4721$, omission: effect of light during $5 \mathrm{C}$ task $P=0.6911$, during $2 \mathrm{C}$ task $P=0.9702$, accuracy\%: effect of light $P=0.2600)$. Of note, our recent study showed that LED illumination over VIS to control mice expressing eYFP in ACA induced no behavioral effects during 5CSRTT (Norman et al., 2021b), suggesting that behavioral changes depend on halorhodopsin expression and are unlikely to be due to other factors such as light induced heat change. It should be though noted that eYFP control experiments were not performed during 2CSRTT. ACAVIS terminal inhibition during any time point examined had no effect on reward collection latency (Supplementary Figure 2A: $t$-test, ITI -5:-2 s $P=0.3846$, ITI $-3: 0$ s $P=0.7773$, stimulus period $P=0.9072$ ), CRL (Supplementary Figure 2B: $t$-test, ITI -5:-2 s $P=0.7012$, ITI $-3: 0$ s $P=0.2568$, during stimulus period $P=0.7620$ ), premature responses (Supplementary Figure 2C: $t$-test, ITI $-5:-2$ s $P=0.1002)$, or perseverative responses (Supplementary Figure 2D: $t$-test, ITI $-5:-2 \mathrm{~s}$ $P=0.5593$, ITI $-3: 0$ s $P=0.5554$, stimulus period $P=0.2756$ ) during the 2CSRTT.

Taken together, these data suggests that ACAVIS projections are causally important for attentional task performance in the period just before stimulus presentation during 5CSRTT, but become dispensable once the task switch to 2CSRTT, suggesting that ACAVIS projections are necessary only when task demand is high.

\section{DISCUSSION}

In this study, we first characterized how attentional behavior outcomes depend on the number of locations for mice to scan and touch for successful performance, and found that the 2CSRTT is less task demanding than the 5CSRTT. We then used circuitspecific optogenetic suppression to show that frontal-sensory $\mathrm{ACA}_{\mathrm{VIS}}$ projection neurons are only crucial for top-down control of attention behavior in male mice during high task demand conditions. These findings support a model that the frontalsensory $\mathrm{ACA}_{\mathrm{VIS}}$ projection regulates visual attention behavior during specific high task demand conditions. Previous studies in mice revealed a causal role of a specific sub-population ACA neurons projecting to the visual cortex in contributing to attentional behavior (Norman et al., 2021a,b), similar to other ACA neurons (Kim et al., 2016; White et al., 2018). However, it remained unclear whether this mechanism was dependent on task demand. Our study provides further insight into defining the role of the specific factor of task demand has in recruiting particular subtypes of neurons.

There are some important limitations with the current study which warrant future investigations. While we showed that prior 5CSRTT training did not affect 2CSRTT performance, one limitation of the current study is that all mice in optogenetic experiments performed the 5CSRTT prior to the 2CSRTT. Future studies should randomize the order in which mice are trained on each of the task in order to control for the contribution of ACAVIS neurons to attentional behavior. It would also informative to investigate the activity of ACAvis neurons during 2CSRTT and 5CSRTT by combining in vivo electrophysiology to examine to what extent ACAvis neuron activity reflects task demand. It should be also noted that this study manipulated a number of choice locations between tasks, but not other parameters which can also impact task demand such as the duration of ITI or visual stimulus. Future studies are warranted to examine to what extent task demand-dependent aspect of ACA $A_{V I S}$ function is generalizable.

Our study provides insight into the neural mechanisms underlying cognitive behavior deficits in psychiatric disease. In low attentional load conditions, attention function while ignoring visual distractors does not differ between patients with schizophrenia and healthy controls but those with schizophrenia performed worse during high load conditions, suggesting that attentional deficits only emerge under more challenging tasks (Ducato et al., 2008). Our study may ultimately inspire consideration of task demand when designing and interpreting 
the results of interventions specifically targeting top-down frontal-sensory circuits to improve attention.

\section{DATA AVAILABILITY STATEMENT}

The raw data supporting the conclusions of this article will be made available by the authors, without undue reservation.

\section{ETHICS STATEMENT}

The animal study was reviewed and approved by the Institutional Animal Care and Use Committee at Icahn School of Medicine at Mount Sinai.

\section{AUTHOR CONTRIBUTIONS}

$\mathrm{KN}$ and HM designed the experiments. $\mathrm{KN}$ and $\mathrm{HM}$ wrote the manuscript with contributions from all co-authors. $\mathrm{KN}$ performed behavioral and optogenetics experiments, and analyzed data with assistance from JB, HK, and TN. JB, PM,

\section{REFERENCES}

Bari, A., Dalley, J. W., and Robbins, T. W. (2008). The application of the 5choice serial reaction time task for the assessment of visual attentional processes and impulse control in rats. Nat. Protoc. 3, 759-767. doi: 10.1038/nprot. 2008.41

Bussey, T. J., Holmes, A., Lyon, L., Mar, A. C., McAllister, K. A., Nithianantharajah, J., et al. (2012). New translational assays for preclinical modelling of cognition in schizophrenia: the touchscreen testing method for mice and rats. Neuropharmacology 62, 1191-1203. doi: 10.1016/j.neuropharm.2011. 04.011

Carli, M., Robbins, T. W., Evenden, J. L., and Everitt, B. J. (1983). Effects of lesions to ascending noradrenergic neurones on performance of a 5-choice serial reaction task in rats; implications for theories of dorsal noradrenergic bundle function based on selective attention and arousal. Behav. Brain Res. 9, 361-380.

Chudasama, Y., Passetti, F., Rhodes, S. E., Lopian, D., Desai, A., and Robbins, T. W. (2003). Dissociable aspects of performance on the 5choice serial reaction time task following lesions of the dorsal anterior cingulate, infralimbic and orbitofrontal cortex in the rat: differential effects on selectivity, impulsivity and compulsivity. Behav. Brain Res. 146, 105-119.

Dalley, J. W., Cardinal, R. N., and Robbins, T. W. (2004). Prefrontal executive and cognitive functions in rodents: neural and neurochemical substrates. Neurosci. Biobehav. Rev. 28, 771-784. doi: 10.1016/j.neubiorev.2004.09. 006

Dillon, G. M., Shelton, D., McKinney, A. P., Caniga, M., Marcus, J. N., Ferguson, M. T., et al. (2009). Prefrontal cortex lesions and scopolamine impair attention performance of C57BL/6 mice in a novel 2-choice visual discrimination task. Behav. Brain Res. 204, 67-76. doi: 10.1016/j.bbr.2009.04. 036

Ducato, M. G., Thomas, P., Monestes, J. L., Despretz, P., and Boucart, M. (2008). Attentional capture in schizophrenia and schizotypy: effect of attentional load. Cogn. Neuropsychiatry 13, 89-111. doi: 10.1080/13546800701707371

Kim, H., Ahrlund-Richter, S., Wang, X., Deisseroth, K., and Carlen, M. (2016). Prefrontal Parvalbumin Neurons in Control of Attention. Cell 164, 208-218. doi: 10.1016/j.cell.2015.11.038
$\mathrm{CC}$, and $\mathrm{KC}$ performed behavioral training. HM supervised the research. All authors contributed to the article and approved the submitted version.

\section{FUNDING}

This work was funded by the NIH F31MH121010 to KN, NIH R21NS105119, R21MH106919, R01EY024918, and R01MH119523 to HM.

\section{ACKNOWLEDGMENTS}

We thank Mark Baxter and Peter Rudebeck for feedback on behavioral and statistical analysis. We also thank members of the Morishita lab for helpful feedback.

\section{SUPPLEMENTARY MATERIAL}

The Supplementary Material for this article can be found online at: https://www.frontiersin.org/articles/10.3389/fnins. 2021.775256/full\#supplementary-material

Koike, H., Demars, M. P., Short, J. A., Nabel, E. M., Akbarian, S., Baxter, M. G., et al. (2016). Chemogenetic Inactivation of Dorsal Anterior Cingulate Cortex Neurons Disrupts Attentional Behavior in Mouse. Neuropsychopharmacology 41, 1014-1023. doi: 10.1038/npp.2015. 229

Lustig, C., Kozak, R., Sarter, M., Young, J. W., and Robbins, T. W. (2013). CNTRICS final animal model task selection: control of attention. Neurosci. Biobehav. Rev. 37, 2099-2110. doi: 10.1016/j.neubiorev.2012.05. 009

Mar, A. C., Horner, A. E., Nilsson, S. R., Alsio, J., Kent, B. A., Kim, C. H., et al. (2013). The touchscreen operant platform for assessing executive function in rats and mice. Nat. Protoc. 8, 1985-2005. doi: 10.1038/nprot. 2013.123

Moore, T., and Zirnsak, M. (2017). Neural Mechanisms of Selective Visual Attention. Annu. Rev. Psychol. 68, 47-72. doi: 10.1146/annurev-psych-122414033400

Norman, K. J., Koike, H., McCraney, S. E., Garkun, Y., Bateh, J., Falk, E. N., et al. (2021a). Chemogenetic suppression of anterior cingulate cortical neurons projecting to the visual cortex disrupts attentional behavior in mice. Neuropsychopharmacol. Rep. 41, 207-214. doi: 10.1002/npr2.12 176

Norman, K. J., Riceberg, J. S., Koike, H., Bateh, J., McCraney, S. E., Caro, K., et al. (2021b). Post-error recruitment of frontal sensory cortical projections promotes attention in mice. Neuron 109, 1202-1213.e5. doi: 10.1016/j.neuron.2021. 02.001

Passetti, F., Chudasama, Y., and Robbins, T. W. (2002). The frontal cortex of the rat and visual attentional performance: dissociable functions of distinct medial prefrontal subregions. Cereb Cortex 12, 1254-1268.

Pehrson, A. L., Bondi, C. O., Totah, N. K., and Moghaddam, B. (2013). The influence of NMDA and GABA(A) receptors and glutamic acid decarboxylase (GAD) activity on attention. Psychopharmacology 225, 31-39. doi: 10.1007/ s00213-012-2792-z

van Gaalen, M. M., Unger, L., Jongen-Relo, A. L., Schoemaker, H., and Gross, G. (2009). Amphetamine decreases behavioral inhibition by stimulation of dopamine D2, but not D3, receptors. Behav. Pharmacol. 20, 484-491. doi: 10.1097/FBP.0b013e3283305e3b 
White, M. G., Panicker, M., Mu, C., Carter, A. M., Roberts, B. M., Dharmasri, P. A., et al. (2018). Anterior Cingulate Cortex Input to the Claustrum Is Required for Top-Down Action Control. Cell Rep. 22, 84-95. doi: 10.1016/j.celrep.2017.12. 023

Zhang, S., Xu, M., Kamigaki, T., Hoang Do, J. P., Chang, W. C., Jenvay, S., et al. (2014). Selective attention. Long-range and local circuits for top-down modulation of visual cortex processing. Science $345, \quad 660-665$. doi: 10.1126/science. 1254 126

Conflict of Interest: The authors declare that the research was conducted in the absence of any commercial or financial relationships that could be construed as a potential conflict of interest.
Publisher's Note: All claims expressed in this article are solely those of the authors and do not necessarily represent those of their affiliated organizations, or those of the publisher, the editors and the reviewers. Any product that may be evaluated in this article, or claim that may be made by its manufacturer, is not guaranteed or endorsed by the publisher.

Copyright () 2022 Norman, Bateh, Maccario, Cho, Caro, Nishioka, Koike and Morishita. This is an open-access article distributed under the terms of the Creative Commons Attribution License (CC BY). The use, distribution or reproduction in other forums is permitted, provided the original author(s) and the copyright owner(s) are credited and that the original publication in this journal is cited, in accordance with accepted academic practice. No use, distribution or reproduction is permitted which does not comply with these terms. 